\begin{tabular}{|c|c|}
\hline Title & $\begin{array}{l}\text { Structural parameter dependence of directed current generation in GaA s nanowire based electron Brownian ratchet } \\
\text { devices }\end{array}$ \\
\hline Author(s) & A be, Y ushi; Kuroda, Ryota; Y ing, X iang; Sato, Masaki; Tanaka, Takay uki; Kasai, Seiya \\
\hline Citation & $\begin{array}{l}\text { Japanese Journal of A pplied Physics(JJA P), 54(6S1), 06FG02 } \\
\text { https://doi.org/10.7567/JJAP.54.06FG02 }\end{array}$ \\
\hline Issue Date & $2015-06$ \\
\hline Doc URL & http:/hdl.handle.net/2115/66632 \\
\hline Rights & (c) 2015 The Japan Society of A pplied Physics \\
\hline Type & article (author version) \\
\hline File Information & JJAP2015_54_MN14088.pdf \\
\hline
\end{tabular}

Instructions for use 


\title{
Structural parameter dependence of directed current generation in GaAs nanowire-based electron brownian ratchet devices
}

\author{
Yushi Abe, Ryota Kuroda, Xiang Ying, Masaki Sato, Takayuki Tanaka, and Seiya Kasai* \\ Research Center for Integrated Quantum Electronics, and Graduate School of Information \\ Science and Technology, Hokkaido University, Sapporo 060-8628, Japan \\ *E-mail: kasai@ rciqe.hokudai.ac.jp
}

We investigated structural parameter dependence of the directed current in GaAs nanowire-based Brownian ratchet devices. The directed current was generated by flashing the ratchet potential array repeatedly using multiple asymmetric gates with a periodic signal. The amount of current in the fabricated device was increased as the nanowire width $W$ was decreased, which contradicted to the theoretical model. The current also depended on the number of the gates $N$, when $N$ was smaller than 6 . We discussed the obtained results in terms of the structure dependence of the carrier transfer efficiency and the effect of the electron reservoirs to the current generation in flashing ratchet operation. 


\section{Introduction}

Biological systems are highly energy efficient as compared to artificial electronic systems. ${ }^{1)}$ Understanding its origin and electronic implementation has been an attractive issues toward ultra low-power electronics. In this point of view, a molecular motor is one of the important examples, which drives muscular contraction exploiting fluctuation. ${ }^{2-5)}$ Its energy conversion efficiency is considered to be as high as $50 \%$. ${ }^{6}$ The mechanism of the molecular motor is known as Brownian ratchet, ${ }^{7,8)}$ in which the motion of Brownian particles is rectified using a periodic array of asymmetric ratchet potentials. ${ }^{9,10)}$ The Brownian ratchet has been artificially demonstrated using various systems such as microparticles, ${ }^{11-17)}$ living cells, ${ }^{18)}$ and electrons. ${ }^{19-23)}$ So far several electron Brownian ratchet devices that generated directed current was reported, ${ }^{19-23)}$ however, the main interest has been in the experimental demonstration and in physical aspect. Next step is to achieve high energy efficiency comparable to the biological system for application. Recently we fabricated the electron Brownian ratchet device using an etched GaAs nanowire with multiple asymmetric Schottky wrap gates and demonstrated the generation of the directed current at room temperature. ${ }^{24)}$ The advantages of this device in terms of the efficiency are collimation of the electron motion into the nanowire direction, and formation and flashing of the ratchet potential by a asymmetric wrap gate that provides tight potential control owing to three-dimensional gate configuration. ${ }^{25-28)}$ In this paper we investigate structural parameter dependence of the directed current in the GaAs-based nanowire Brownian ratchet device. We fabricate and characterize the devices having various nanowire width and number of gates in terms of the amount of the current and the carrier transfer efficiency.

\section{Concept and experiment}

The mechanism of the Brownian ratchet is schematically shown in Fig. 1(a). In case of the biological molecular motor, the actin filament is the one-dimensional rail for running myosin. The chemical interaction between the actin and myosin realizes the situation as shown in Fig. 1(a). ${ }^{3,9)}$ In case of the electron Brownian ratchet, the ratchet is electrically formed with an asymmetric electrostatic potential. Consider that the electrons with Brownian motion are accumulated in the potential valleys as shown in the top of Fig. 
1(a). Then the potential is disappeared, the electrons move forward and backward equally due to thermal diffusion. When the ratchet potential is formed again, a part of the diffusing electrons moves to the left next valley whereas the others are again trapped in the same valley in the previous step. Repeating this process, directed electron flow is generated. This type of operation is called as "flashing ratchet".

A GaAs nanowire-based Brownian ratchet device with a measurement setup is schematically shown in Fig. 1(b). The device has multiple wedge-shaped Schottky wrap gates on a GaAs-based nanowire. A ratchet potential is formed underneath an asymmetric gate. The top of the ratchet potential locates under the top of the wedge in the gate. The potential height depends on gate voltage $V_{\mathrm{G}}$. Flashing of the potentials is achieved by applying a periodic voltage signal to the gates. The GaAs nanowire was formed on a modulation-doped $\mathrm{AlGaAs} / \mathrm{GaAs}$ heterostructure by electron beam lithography and wet chemical etching. Figure 1(c) shows a scanning electron microscope image of a fabricated device. Mobility $\mu$ and sheet carrier concentration $n_{\mathrm{S}}$ of the two-dimensional electron gas (2DEG) were $7,100 \mathrm{~cm}^{2} \cdot \mathrm{V}^{-1} \cdot \mathrm{s}^{-1}$ and $7.8 \times 10^{11} \mathrm{~cm}^{-2}$, respectively, at room temperature. Measured gate threshold voltage of the fabricated devices was $0.3 \mathrm{~V}$, almost not depending on the device dimension. For the flashing ratchet operation, all gates were biased at $0 \mathrm{~V}$ and a square wave with a peak-to-peak voltage $V_{\mathrm{PP}}$ of $1 \mathrm{~V}$ was applied simultaneously. In this condition, the input signal crossed the threshold voltage; the ratchet potential was disappeared (OFF potential) when $V_{\mathrm{G}}=0.5 \mathrm{~V}$, and the ratchet potential was formed (ON potential) when $V_{\mathrm{G}}=-0.5 \mathrm{~V}$. DC net current $I_{\mathrm{NET}}$ in the nanowire was measured using a conventional semiconductor parameter analyzer without biasing the nanowire as shown in Fig. 1(b). In this study, we characterized nanowire width dependence and number of gates dependence of $I_{\text {NET }}$. Fabricated device dimensions are summarized in Table I. The nanowire width $W$ was varied from 300 to $720 \mathrm{~nm}$. The gate length $L_{\mathrm{G}}$ and the distance between the gates $L_{\mathrm{D}}$ were around $300 \mathrm{~nm}$ and $130 \mathrm{~nm}$, respectively. The top angle of the wedge in the Schottky wrap gate was $90^{\circ}$ and the length of the narrowest part of the gate was $130 \mathrm{~nm}$. The number of the gates was varied from 2 to 20. All measurements were carried out at room temperature.

\section{Results and discussion}




\subsection{Nanowire width dependence}

Figure 2(a) shows the measured net current $I_{\mathrm{NET}}$ as a function of flashing frequency $f$. The nanowire widths of the measured devices were $300,500,680$, and $720 \mathrm{~nm}$. The negative current was generated when the flashing frequency was higher than $1 \mathrm{MHz}$ and the amount of current increased as the frequency increased. The polarity of the current agreed with the ideal one that was determined from the ratchet potential configuration. The observed frequency dependency of the current suggested that it was neither the gate leakage current nor the rectification of the input signal through the Schottky gate; these currents do not depend on the input signal frequency. We also found that the current increased as the nanowire width decreased. This dependency contradicted the usual size dependence of the resistance.

We compare the experimental $I_{\mathrm{NET}}-f$ curve with the theoretical one. $I_{\mathrm{NET}}$ is calculated from a simple model expressed by ${ }^{29,30)}$

$$
I_{N E T}=\beta \times L W \times e n_{s} \times f,
$$

where $L=L_{\mathrm{G}}+L_{\mathrm{D}}, \beta$ is the carrier transfer rate corresponding to the fraction of the carriers moving to the next potential valley and $e$ is the elemental charge. In this model parameters excepting $\beta$ are known. At most half of the electrons contribute to the net current, thus the maximum value of $\beta$ is $0.5 .^{30}$ The theoretical curves for the devices with the same dimensions in Fig. 2(a) are shown in Fig. 2(b). Theoretically the current increases as $W$ increases. Experimentally obtained $W$ dependence also contradicts the theory.

To understand the obtained $W$ dependence of $I_{\mathrm{NET}}$, we consider that the carrier transfer rate itself depends on the nanowire width. Then the effective carrier transfer rate $\beta_{\text {eff }}$ is estimated from the experimental data using the equation,

$$
\beta_{\text {eff }}=\frac{I_{N E T}}{L W \times e n_{s} \times f} .
$$

The evaluated $\beta_{\text {eff }}$ is plotted in Fig. 3. $\beta_{\text {eff }}$ shows a linear dependence on $W$ and it increases as $W$ decreases. This suggests that the experimentally observed $W$ dependence is caused by 
the $W$ dependence of the ratchet potential configuration. For confirmation, we carried out the potential simulation by numerically solving the three-dimensional Poisson equation. Figure 4 shows the obtained ratchet potential in the channel at $V_{\mathrm{G}}=-0.5 \mathrm{~V}$. Gray hatched area represents the gate electrode region. Height and width of the ratchet potential become large as $W$ decreases. Simultaneously symmetry of the potential changes depending on $W$; the potential shape is rather asymmetric when $W=200 \mathrm{~nm}$, whereas it is almost symmetric when $W=500 \mathrm{~nm}$. This is because the combination of the electric fields from the Schottky gate on the nanowire top and sidewalls determines the ratchet potential configuration. The simulation results indicate that the experimentally observed $W$ dependence of $I_{\text {NET }}$ arises from the $W$ dependence of the ratchet potential asymmetry. If the ratchet potential is completely symmetric, the amounts of electrons moving forward and backward are not biased, and the directed current flow is not generated.

For quantitative discussion on the relationship between $\beta$ and $W$, we estimate $\beta$ focusing on the distance between the neighboring potentials $L$ - $M$, where $M$ is the ratchet potential width. Electrons in the flat band region at $V_{\mathrm{G}}=-0.5 \mathrm{~V}$ do not contribute to the directed current. Thus $\beta$ should decrease as the flat band region is extended. $\beta_{\text {eff }}$ considering this effect is expressed by $(M / L) \beta_{0}$, where $\beta_{0}$ is the maximum carrier transfer efficiency at $M=L$. Approximating the ratchet potential in Fig. 4 by a complete saw tooth, then $\beta_{0}$ is given by $0.5 \operatorname{erfc}\left(M / \sqrt{2 \sigma^{2}+2 D_{e} / f}\right)$, where $\operatorname{erfc}$ is the complementary error function, $\sigma$ is the standard deviation of the thermally distributed electrons in space, and $D_{\mathrm{e}}$ is the electron diffusion coefficient. ${ }^{30)}$ In our device, the diffusion length is estimated to be enough longer than $M$, thus $\beta_{0}=0.5$ is expected. The potential simulation results in Fig. 4 show that $M$ increases as $W$ decreases at the same $V_{\mathrm{G}}$ condition. It is noted that the ratchet potential configuration in the nanowire direction depends on its lateral position as shown in the inset of Fig. 4, which is an example of the two-dimensional potential distribution in the 2DEG layer. Therefore, for calculating $\beta_{\text {eff }}$, we use averaged $M$ in the lateral direction. The calculated $\beta_{\text {eff }}$ is also plotted in Fig. 3. The model reproduces the monotonically decreasing curve similar to the experimental one. The observed discrepancy indicates that the simulated ratchet potential is smaller than the experimental one. This mainly arises from the threshold voltage difference between the simulation and the experiment, unexpectedly 
caused even though the nominally same device parameters are used in the simulation. By carefully adjusting the parameters in simulation, the model will explain the experimental $\beta_{\text {eff. }}$ Further study is necessary for more quantitative understanding of the obtained behavior. Another information from the model is the possibility of increasing the carrier transfer efficiency by further decreasing the nanowire width. This is an important design guideline for high efficiency of the device.

\subsection{Dependence of number of gates}

Measured flashing ratchet current in the fabricated devices having a different number of gates $N$ and estimated $\beta_{\text {eff }}$ are shown in Figs. 5(a) and 5(b), respectively. The number of gates was $2,5,6$, and 20 . When $N$ was less than 6 , the current decreased with decreasing $N$, whereas the current did not depend on $N$ when $N$ was larger than 6 . Similarly, the effective carrier transfer rate for $N \geq 6$ was constant approximately at $30 \%$, whereas it decreased to $0 \%$ as decreasing $N$ to 2 when $N<6$. The result shows that sufficient number of gates is necessary to generate a directed current.

We consider this requirement in terms of the effect of the electron reservoirs. Here the bare nanowire portions connecting to the ends of the ratchet potential array are considered to work as electron reservoirs. Then Fermi level $E_{\mathrm{F}}$ in the ratchet portion near the reservoir is in thermal equilibrium to that of the reservoir, since a sufficient number of electrons can be promptly supplied from the reservoir. If the length of the ratchet potential array $N \cdot L$ is enough shorter than the electron diffusion length $\lambda_{\mathrm{e}}, N \cdot L<2 \lambda_{\mathrm{e}}$, Fermi level through the ratchet potential array should be in equilibrium to that of the reservoirs, even in flashing operation. This situation suggests that the device cannot make any DC current flow and the carrier transfer efficiency decreases. On the other hand, when $N \cdot L>2 \lambda_{\mathrm{e}}$, the amount of electrons diffused from the reservoirs are attenuated in the middle of the ratchet potential array. Then electrons in the ratchet potential locally are conserved and $E_{\mathrm{F}}$ is isolated from that of the reservoirs. In this case, by transferring the electrons with the flashing operation, the device is possible to produce the electrostatic potential difference inside and could generate DC current driving the external circuit.

We estimate the carrier diffusion length in our device. Here we assume that the carrier lifetime $\tau_{\mathrm{e}}$ is dominated by the surface recombination because of the high-density surface states of the GaAs surface together with high surface-to-bulk ratio of the 
nanowire. ${ }^{31)} \tau_{\mathrm{e}}$ is evaluated by $\tau_{\mathrm{e}}=l / v_{\mathrm{SS}}$, where $l$ is the distance between 2DEG channel and surface and $v_{\mathrm{SS}}$ is surface recombination velocity. $v_{\mathrm{SS}}$ on GaAs surface is approximately $10^{5} \mathrm{~cm} \cdot \mathrm{s}^{-1} \cdot{ }^{31,32)}$ Diffusion length is estimated by $\lambda_{\mathrm{e}}=\left(D_{\mathrm{e}} \tau_{\mathrm{e}}\right)^{1 / 2}$, where $D_{\mathrm{e}}$ is electron diffusion coefficient, and we obtain $\lambda_{\mathrm{e}}=1 \mu \mathrm{m}$. The model suggests that the current is decreased and $\beta_{\text {eff }}$ is reduced when $N \cdot L<2 \lambda_{\mathrm{e}}$, otherwise the these do not depend on $N$. From $L=L_{\mathrm{G}}+L_{\mathrm{D}}=400 \mathrm{~nm}$ in our device, the model indicates that the current is decreases when $N \leq 5$, which reasonably explains the behavior in Fig. 5(b). The model with the discussion suggests the effect of the electron reservoirs on the Brownian ratchet device and provides a design guideline in terms of the device length, the gate length, and the number of the gates.

\section{Conclusions}

Structural parameter dependence of the directed current in GaAs nanowire-based Brownian ratchet devices was experimentally investigated for optimizing the device design. The current was increased as the nanowire was narrowed from $720 \mathrm{~nm}$ to $300 \mathrm{~nm}$. Lateral depletion from the side of the nanowire enlarged the ratchet potential, which improved the carrier transfer rate. The directed current was found to also depend on the number of the asymmetric gates. This result indicated that the ratchet potential array needed to have sufficient length to attenuate the carrier diffusion from the electron reservoirs for current generation by flashing ratchet operation.

\section{Acknowledgments}

This work was partly supported by Grant-in-Aid for Scientific Research on Innovative Areas "Molecular Architectonics: Orchestration of Single Molecules for Novel Functions" (No. 25110001). 


\section{References}

1) R. Sarpeshkar: Ultra Low Power Bioelectronics (Cambridge University Press, NewYook, 2010) Sect. VI.

2) C. Bustamante, Y. R. Chemla, N. R. Forde, and D. Izhaky: Annu. Rev. Biochem. 73, 705 (2004).

3) T. Yanagida and Y. Ishii: J. Muscle Res. Cell Motility 33, 395 (2013).

4) M. O. Magnasco: Phys. Rev. Lett. 72, 2656 (1994).

5) R. A. Haddou and W. Herzog: Cell Biochem. Biophys. 38, 191 (2003).

6) H. Tanaka, K. Homma, A. Hikikoshi Iwane, E. Katayama, R. Ikebe, J. Saito, T. Yanagida, and M. Ikebe: Nature 415, 193 (2002).

7) P. Hanggi and F. Marchesoni: Rev. Mod. Phys. 81,387 (2009).

8) R. P. Feynman, R. B. Leighton, and M. Sands: The Feynman Lectures on Physics (Addison-Wesley, Reading, MA, 1996) Vol. 1.

9) R. D. Astumian and M. Bier: Phys. Rev. Lett. 72, 1766 (1994).

10) T. Salger, S. Kling, T. Hecking, C. Geckeler, L. M. Molina, and M. Weitz: Science 326, 1241 (2009).

11) T. Motegi, H. Nabika, and K. Murakoshi: Langmuir 28, 6656 (2012).

12) V. A. Oudenaarden and S. G. Boxer: Science 285, 1046 (1999).

13) B. J. Lopez, N. J. Kuwada, E. M. Craig, B. R. Long, and H. Linke: Phys. Rev. Lett. 101, 220601 (2008).

14) P. Tierno, P. Reimann, T. H. Johansen and F. Sagues: Phys. Rev. Lett. 105, 230602 (2010).

15) L. Gao, M. A. Tahir, L. N. Virgin, and B. B. Yellen: Lab Chip 11, 4214 (2011).

16) J. Rousselet, L. Salome, A. Ajdari, and J. Prostt: Nature 370, 446 (1994).

17) H. S. Elizabeth, W. R. Diluzio, S. S. Shevkoplyas, L. Turner, M. Mayer, H. C. Berg, and G. M. Whitesides: Lab Chip 8, 1888 (2008).

18) G. Mahmud, C.J. Campbell, K. J. M. Bishop, Y. A. Komarova, O. Chaga, S.Soh, S. Huda, K. Kandere- Grzybowska, and B. A. Grzybowski: Nat. Phys. 5, 606 (2009).

19) A. Lorke, S. Wimmer, B. Jager, J. P. Kotthaus, W. Wegscheider, and M. Bichler: Physica B 251, 312 (1998). 
20) H. Linke, T. E. Humphrey, P. E. Lindelof, A. Löfgren, R. Newbury, P. Omling, A. O. Sushkov, R. P. Taylor, and H. Xu: Appl. Phys. A 75, 237 (2002).

21) E. M. Roeling, W. C. Germs, B. Smalbrugge, E. J. Geluk, T. D. Vries, R. A. J. Janssen, and M. Kemerink: Nat. Mater. 10, 51 (2011).

22) S. Sassine, Y. Krupko, J. C. Portal, Z. D. Kvon, R. Murali, K. P. Martin, G. Hill, and A. D. Wieck: Phys. Rev. B 78, 045431 (2008).

23) A. M. Song, P. Omling, L. Samuelson, W. Seifert, I. Shorubalko, and H. Zirath: Appl. Phys. Lett. 79, 1357 (2001).

24) T. Tanaka, Y. Nakano, and S. Kasai: Jpn. J. Appl. Phys. 52, 06GE07 (2013).

25) S. Kasai, K. Jinushi, T. Tomozawa, and H. Hasegawa: Jpn. J. Appl. Phys. 36, 1678 (1997).

26) M. Yumoto, S. Kasai, and H. Hasegawa: Jpn. J. Appl. Phys. 41, 2671 (2002).

27) M. Yumoto, S. Kasai, and H. Hasegawa: Microelectron. Eng. 63, 287 (2002).

28) H.-Q. Zhao, S. Kasai, Y. Shiratori, and H. Hashizume, Nanotechnology. 20, 245203 (2009).

29) J. Rousselet, L. Salome, A. Ajdari and J. Prostt: Nature 370, 446 (1994).

30) M. Kabir, D. Unluer, L. Li, A. W. Ghosh, and M. R. Stan: IEEE Trans. Nanotechnol. 12, 330 (2013).

31) H. J. Joyce, C. J. Docherty, Q. Gao, H. H. Tan, C. Jagadish, J. Lloyd-Hughes,

L. M. Herz, and M. B. Johnston: Nanotechnology. 24, 214006 (2013).

32) D. E. Aspnes: Surf. Sci. 132, 406 (1983). 


\section{Table Caption}

Table I. Structural parameters of the fabricated devices. $W$ is nanowire width, $L_{\mathrm{G}}$ is gate length, and $L_{\mathrm{D}}$ is distance between the gates.

\section{Figure Captions}

Fig. 1. (Color online) (a) Mechanism of Brownian ratchet in case of flashing operation, (b) schematic illustration of a GaAs nanowire-based Brownian ratchet device with measurement circuit, and (c) a scanning electron microscope image of a fabricated device.

Fig. 2. (Color online) (a) Measured $I_{\mathrm{NET}}$ as a function of flashing frequency for the devices having various nanowire widths. (b) Theoretical $I_{\mathrm{NET}}-f$ curves for the device with the same dimensions.

Fig. 3. (Color online) Evaluated effective carrier transfer rate $\beta_{\text {eff }}$ as a function of nanowire width. Theoretical curve is also shown.

Fig. 4. (Color online) Ratchet potentials at $V_{\mathrm{G}}=-0.5 \mathrm{~V}$ for various nanowire widths obtained from the three-dimensional potential simulation. The inset shows an example of the potential map in $2 \mathrm{DEG}$ plane.

Fig. 5. (Color online) (a) Measured $I_{\mathrm{NET}}-f$ curves for the devices having various numbers of gates $N$ and (b) evaluated $\beta_{\text {eff }}$ as a function of $N$. 